\title{
Persistência da artéria isquiática: relato de caso
}

\author{
Persistent sciatic artery: a case report \\ Leonardo G hizoni Bez¹, Ricardo C osta-Val2, Paulo Bastianetto ${ }^{3}$, \\ Tatiana Ferreira de 0 liveira ${ }^{3}, \mathrm{C}$ arlos Eduardo M ourad Avelar ${ }^{3}$, \\ Denise Sena Veloso30 ${ }^{3}$ Jovelino Pinheiro C osta³, Fábio Sales de O liveira
}

\begin{abstract}
Resumo
A persistência da artéria isquiática é uma anomalia congênita rara do sistema circulatório, sendo descritos poucos casos na literatura e não existindo, assim, consenso sobre métodos diagnósticos e de tratamento. 0 s autores apresentam o caso de um paciente masculino, de 59 anos de idade, com história de dor tipo "claudicação intermitente" progressiva em panturrilha direita, associada a uma massa pulsátil em fossa poplítea e ausência de pulsos distais. A angiotomografia da aorta abdominal e dos membros inferiores revelou persistência da artéria isquiática à direita, sendo realizada, ainda, angiografia com subtração digital seletiva da artéria isquiática para o planejamento cirúrgico. 0 paciente foi submetido à ponte fêmoro-fibular com veia safena magna ipsolateral reversa eligadura da artéria isquiática na pelve, por acesso retroperitoneal.
\end{abstract}

Palavras-chave: Persistência da artéria isquiática, aneurisma da artéria isquiática, terapêutica cirúrgica, isquemia periférica.

\section{Introdução}

A persistência da artéria isquiática (PAI) é uma anomalia vascular congênita rara, com prevalência de 0,01-0,05\% na população, sendo descritapela primeira vez por G reen em 1832 e demonstrada angiograficamente por Cowie et al. em 19601,2.

1. Coordenador, Serviço de Angiologia e Cirurgia Vascular, Instituto de Previdência dos Servidores do Estado de Minas Gerais (IPSEMG), Belo Horizonte, MG. Membro efetivo, SBACV. Especialista em Angiologia, Cirurgia Vascular e Endovascular, SBACV.

2. Membro titular, SBACV. Mestre e Doutor em Cirurgia, Universidade Federal de Minas Gerais (UFMG), Belo Horizonte, MG. Professor, Graduação e Pós-Graduação, Universidade Vale do Rio Verde (UNINCOR), Belo Horizonte, MG

3. Residente de Angiologia e Cirurgia Vascular, IPSEMG, Belo Horizonte, MG.

Artigo submetido em 20.06.06, aceito em 30.08.06.

Copyright $\odot 2006$ by Sociedade Brasileira de Angiologia e de Cirurgia Vascular.

\begin{abstract}
Persistent sciatic artery is a rare congenital anomaly of the circulatory system. Sincethereare few cases described in theliterature, there is no consensus on diagnostic and treatment methods. The authors report on a59-year-old male patient with history of progressive intermittent claudication in the right calf, associated with pulsatile mass in the popliteal fossa and absence of distal pulses. Tomography angiography of the abdominal aorta and lower limbs showed persistent right sciatic artery. Selective digital subtraction angiography of the sciatic artery was also performed for surgical planning. The patient was submitted to femorofibular bypass with reverse ipsilateral great saphenous vein and ligation of the sciatic artery in the pelvis using retroperitoneal approach.
\end{abstract}

Key words: Persistent sciatic artery, sciatic artery aneurysm, surgical correction, peripheral ischemia.

Essa artéria anômala está predisposta a degeneração ateromatosa precoce, dilatação aneurismática, tromboembolismo distal e oclusão arterial. Seu diagnóstico é realizado freqüentemente em paciente com quadro clínico de isquemia aguda no membro inferior, complicações tromboembólicas distais, massa glútea palpável, ou como um achado ocasional ao exame clínico, métodos de imagem ou necropsia em pacientes assintomáticos ${ }^{3}$.

0 presente artigo relata um caso de PAI com degeneração aneurismática e complicações isquêmicas no membro inferior direito (MID).

\section{Relato do caso}

Pacientemasculino, com 59 anosdeidadeehistória declaudicação em panturrilha direita ao caminhar para 
cerca de 50 metros. Foi avaliado inicialmente por um ortopedista, que, ao solicitar um ultra-som de partes moles, diagnosticou cisto de Baker e aneurisma de artéria poplítea, sendo encaminhado, então, para avaliação angiológica. 0 dupl ex scan ea arteriografia convencional revelaram oclusão total da artéria femoral superficial, sendo o paciente encaminhado ao Serviço de Angiologia e Cirurgia V ascular do H ospital do Instituto de Previdência dos Servidores do Estado de M inas G erais (IPSEM G).

Em sua história pregressa, não havia outras comorbidades, nem passado detabagismo. Seu exame arterial periférico mostrou-se sem anormalidades à esquerda, e no M ID o pulso femoral era palpável, de baixa amplitude, mas o pulso poplíteo era facilmente palpável, amplo, cheio, rítmico e simétrico. Pal pava-se também facilmente um pulso desde a fossa poplítea atéa nádega direita, por toda a face posterior da coxa. Os pulsos distais não eram palpáveis, e ao Doppler de ondas contínuas foi observado som de padrão monofásico somente na artéria fibular, cujo índice braço/perna era de 0,4 .

Levantada a possibilidade de aneurisma de artéria poplíteaeafim deseexcluir a associação com aneurisma da aorta abdominal eaneurisma no membro oposto, foi solicitada uma angiotomografia (ATC) da aorta abdo- minal e membros inferiores, que revelou, no M ID, a PAI primitiva aneurismática, com trombos em seu interior, arteriomegálica (dilatada e tortuosa) desde sua origem atéa artéria poplítea. Foi realizada também uma angiografia com subtração digital e cateterização seletiva das ilíacas interna e externa para programação cirúrgica, que evidenciou a artéria ilíaca externa e sistema femoral hipoplásicos e presença somente de artéria fibular pérvia na perna direita (Figuras 1 e 2).

Foi proposto tratamento cirúrgico, que se deu por meio de ponte fêmoro-fibular com veia safena magna reversa ipsolateral associada a ligadura da artéria isquiática. A anastomose proximal da ponte foi realizada na artéria femoral superficial, que, apesar de hipoplásica, apresentava um pulso amplo sem evidências macroscópicas de lesões ateromatosas por acesso clássico - provavelmente, a discrepância da amplitude dos pulsos entre o exame clínico e o achado peroperatório se deve ao calibre da artéria e à massa muscular bem desenvolvida do paciente, fazendo com que o pulso ao exame clínico fosse difícil de ser palpado, sendo, então, classificado como de "baixa amplitude". A artéria fibular foi abordada no terço distal da perna, lateral mente, com ressecção de aproximadamente $8 \mathrm{~cm}$ da fíbula. As anastomoses foram confeccionadas em sutura contínua com fio de polipropileno 7-0. Para se al cançar a artéria isquiá-
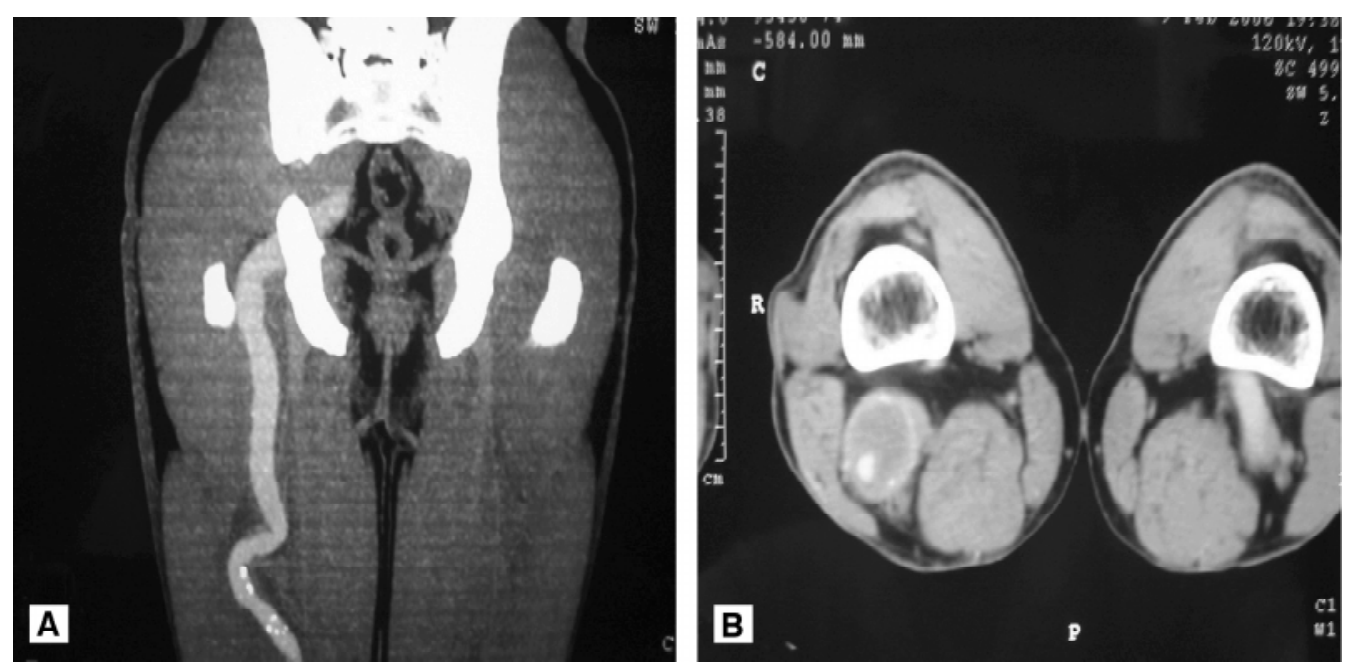

Figura 1 - A) Reconstrução angiotomográfica demonstrando persistência da artéria isquiática primitiva. B) Corte transversal evidenciando degeneração aneurismática com trombos em seu interior 


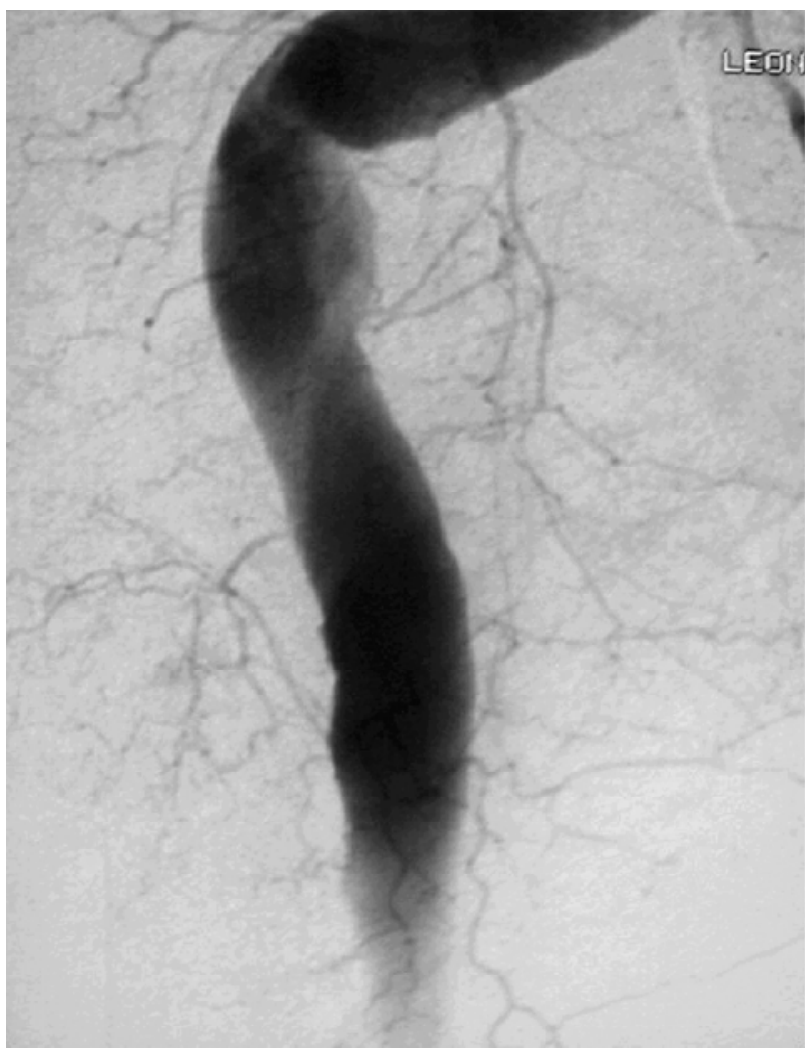

Figura 2 - Angiografia com subtração digital seletiva da artéria isquiática interna

tica primitiva na pelve, utilizamos uma incisão de aproximadamente $13 \mathrm{~cm}$, arqueada, na fossa ilíaca direita, por via retroperitoneal, sendo a ligadura cirúrgica da mesma realizada o mais distalmente possível, após emergência deramo glúteo e da seguintemaneira: ligadura dupla; proximal, com fio de seda; e distal em sutura contínua, com fio de polipropileno 3-0. 0 pósoperatório transcorreu sem anormalidades, e o paciente recebeu alta hospital ar para acompanhamento ambulatorial, assintomático, apresentando índice braço/perna de 0,83 .

\section{D iscussão}

A origem embriológica dessa anomalia vascular apresenta-se bem conhecida. $\mathrm{N}$ o embrião de $9 \mathrm{~mm}$ de comprimento (5 a 6 semanas), a artéria isquiática, ou artéria axial, que se origina da raiz dorsal da artéria umbilical, éo principal ramo que nutre o pé primitivo.
Já no embrião de $10 \mathrm{~mm}$ de comprimento, dá-se início ao desenvolvimento do sistema femoral como continuação da artériailíaca externa, quese di lata eemiteramos para a coxa medial e distal. No embrião de $14 \mathrm{~mm}$ de comprimento (cerca de 8 semanas), o membro inferior tem suprimento sangüíneo duplo, pela artéria isquiática e femoral. D urante o terceiro mês de gestação (cerca de 12 semanas), ocorre atrofia progressiva da artéria isquiática na coxa distal, com desenvolvimento do sistema femoral superficial. N o adulto, os remanescentes da artéria isquiática participam da formação da porção proximal das artérias glúteas superior e inferior, artéria poplítea, origem da tibial anterior, artéria fibular, econtribuem paraanastomosesterminaisno pé $e^{1,2,4}$.

$\mathrm{Em}$ relação à $\mathrm{PAl}$, são descritas duas formas, a saber ${ }^{1,3}$ :

- Forma completa (63-79\% dos casos), onde essa artéria é o principal vaso de suprimento para 0 membro inferior, estando o sistema femoral superficial hipoplásico, mas raramente ausente;

- Forma incompleta (cerca de 20\% dos casos), onde a artéria isquiática encontra-se hipoplásica, comunicando-sepor meio de vários ramos com o sistema femoral, que se apresenta sem anormalidades ${ }^{5-7}$.

$\mathrm{N}$ ão existediferençana incidência quanto a sexo ou idade, sendo descritos casos de recém-nascidos a octogenários, e, em 12\% dos casos, essa anormalidade está presente em ambos os membros inferiores ${ }^{2}$. A artéria anômala acompanha o trajeto do nervo isquiático na região glútea e face posterior da coxa. Sabe-se também que a PAI pode estar associada a outras malformações, como rim único, fístula arteriovenosa, hipertrofia de extremidade, varicosidades, hipertrofia de partes moles, hipertrofia óssea, neurofibromatose e anomalias de outras artérias e veias².

A maioria dos pacientes são sintomáticos (70\%), e as manifestações clínicas estão relacionadas geralmente ao quadro de degeneração aneurismática, que ocorre em $25-58 \%$ dos pacientes. A alta incidência de degeneração aneurismática se deve a microtraumas repetidos na zona glútea e também à hipoplasia de fibras de elastina na parede arterial ${ }^{5,7}$.

D entre as manifestações clínicas mais freqüentes, temos a isquemia aguda, a isquemia crônica (secundárias a eventos tromboembólicos distais), massa glútea pulsátil e sinais de compressão do nervo isquiático ${ }^{3}$.

A presença de alguns sinais clínicos pode orientar a suspeita diagnóstica, tais como a ausência ou diminui- 
ção de pulso femoral, com pulsos poplíteos e distais pal páveis, associados ou não a massa glútea pulsátil ${ }^{8}$.

0 diagnóstico diferencial deve ser feito, principalmente, com aneurisma deartéria glútea, abscessos glúteos, neoplasias, fístula arteriovenosa e lombociatalgias².

A arteriografia com subtração digital éconsiderada o padrão-ouro para o diagnóstico dessa doença. Porém, deve-se ressaltar a importância da cateterização sel etiva das ilíacas internas para 0 adequado estudo da artéria anômala. As artérias ilíacas externas, o sistema femoral e as artérias distais também devem ser avaliados para 0 correto planejamento cirúrgico. Pode-se ter uma impressão errônea acerca da perviedade da artéria poplítea e dos vasos distais, devido ao baixo fluxo na artéria dilatada, o que pode levar a erros na programação terapêutical,8.

Além da arteriografia, a PAI pode ser diagnosticada pela ATC , angiorressonância e duplex scan. Esses exames são capazes de fornecer dados como dimensão e correlação com estruturas anatômicas adjacentes. A ATC pode ser utilizada como método diagnóstico inicial eúnico, revelando relação com tecidos vizinhos, estrutura das partes moles, osso, veias, nervos, outras artérias, a presença de aneurismas, trombose e oclusão total. A PAI apresenta uma relação anatômica constante, sen do vista naAT C como uma continuação tortuosa da artériailíaca interna, queentra na coxa posterior pelo forame isquiático, passa abaixo do músculo glúteo máximo e, continuando pela face posterior do músculo adutor magno, passa dentro da fossa poplítea, continuando como artéria poplítea e estando neste ponto anterior ao nervo isquiático ${ }^{1,9}$.

$\mathrm{N}$ a maioria dos pacientes portadores de $\mathrm{PAI}$, a veia femoral superficial encontra-se hipoplásica, sendo a drenagem venosa feita pela veia ciática persistente ou por uma veia comunicante entre a veia poplítea ea veia femoral profunda (veia comunicante posterior do joeIho - $75 \%$ dos casos) ${ }^{1}$.

0 tratamento desta afeção é controverso, e não há ainda um consenso estabelecido naliteratura. $N$ oscasos da PAI assintomáticos e sem sinais de degeneração aneurismática, o acompanhamento clínico e o seguimento do paciente parecem ser a mel hor opção. Para os pacientes sintomáticos e/ou com sinais de complicações, o tratamento cirúrgico éobrigatório. A revascularização in situ não éaconselhada, pelo risco de lesão do nervo isquiático e pela possível compressão da ponte quando o paciente assumir a posição assentada. Como opções terapêuticas, são descritos: a) exclusão do aneurisma e ponte pelo forame obturador; b) ligadura proximal e distal com ponte fêmoro-poplítea; c) ponte femoral com enxerto; d) excisão da artéria isquiática com interposição deenxerto; e) tratamento endovascular com embolização; f) tratamento endovascular com stent graft $1,3,5$.

\section{Conclusão}

A PAI é uma anomalia rara, deven do fazer partedo diagnóstico diferencial das doençasvascularesdosmembros inferiores. A ATC e a angiografia com subtração digital são excelentesmétodos para diagnóstico eplanejamento cirúrgico. 0 tratamento eo seguimento devem ser individualizados para cada paciente.

\section{R eferências}

1. Jung AY, LeeW, C hungJW , Song SY, Kim SJ, H aJ , et al. Role of computed tomographic angiography in the detection and comprehensive evaluation of persistent sciatic artery. J V asc Surg. 2005;42:678-83.

2. Juliá J, Rimbau EM, Gómez F, Lozano P, Corominas C. Arteria ciática persistente bilateral. Rev Angiol. 1995;4:199205.

3. I shida K, Imamaki M, I shida A, Shimura H , M iyazaki M . A ruptured aneurysm in persistent sciatic artery: a case report. J V asc Surg. 2005;42:556-8.

4. H assan A. Symptomatic persistent sciatic artery. J Am Coll Surg. 2004;199:171-3.

5. Fearing NM, Ammar AD, Hutchinson SA, Lucas ED. Endovascular stent graft repair of a persistent sciatic artery aneurysm. Ann V asc Surg. 2005;19:438-41.

6. I kezawa T, N aiki K, M oriura S, I keda S, H irai M . Aneurysm of bilateral persistent sciatic arteries with ischemic complications: case report and review of the world literature. J V asc Surg. 1994;20:96-103.

7. M athias KD, Feldmuller $M, H$ aarmann $P$, Goldbeck $F$. Persistent sciatic artery: bilateral percutaneous transluminal angioplasty in ischemic disease. Cardiovasc Intervent Radiol. 1993;16:377-9.

8. Samson RH , Showalter D P. Persistent sciatic artery as collateral for an occluded iliofemoral system. J V asc Surg. 2004;40:183.

9. Erturk SM , T atli S. Persistent sciatic artery aneurysm. J V asc Interv Radiol. 2005;16:1407-8.

Correspondência:

Paulo Bastianetto

Rua Engenheiro Amaro Lanari, 389/101

CEP 30310-580 - Belo H orizonte, M G

E-mail: paulobast@yahoo.com.br 